\title{
Diseño y evaluación de un sistema de refrigeración experimental trabajando por adsorción solar
}

\author{
Design and evaluation of an experimental refrigeration system working by solar \\ adsorption
}

\author{
Francisco Ernesto Moreno García $^{1 *} \quad$ Enio Pedone Bandarra filho ${ }^{2} \quad$ Dinael Guevara Ibarra ${ }^{3}$
}

Recibido 7 de marzo de 2017, aceptado 25 de septiembre de 2017

Received: March 7, 2017 Accepted: September 25, 2017

\begin{abstract}
RESUMEN
Este trabajo presenta una caracterización de un sistema de refrigeración empleando la radiación solar como única fuente de energía para generar el fenómeno de la adsorción asistido en un colector estacionario tipo placa plana. Este colector presenta un área de captación de $1.1 \mathrm{~m}^{2}$ trabajando con carbón activado como adsorbato y empleando metanol como adsorbente. Los resultados experimentales permitieron compararse en dos condiciones de trabajo cuya finalidad fue enfriar una pequeña cantidad de agua reservada de 2 litros. Se registraron temperaturas de generación en el colector solar por encima de los $50^{\circ} \mathrm{C}$ para intensidades solares por encima de los 800 [watt $\left./ \mathrm{m}^{2}\right]$. El COP del sistema se presentó por encima de los 0,4 según etapas de enfriamiento y adsorción isobárica. Se realizaron mediciones de radiación solar, se tomaron mediciones de temperatura del sistema en funcionamiento, dando como resultados diferencias de temperatura de hasta $8,6{ }^{\circ} \mathrm{C}$ entre la temperatura ambiente y la temperatura de la recámara de frio. Se tomaron mediciones de radiación local de hasta $1155\left[\mathrm{watt} / \mathrm{m}^{2}\right]$, dando resultados de eficiencia de nuestro colector de hasta $75 \%$ en horas específicas y de $35 \%$ en eficiencia total diaria. Aprovechando herramientas a nivel de diseño de experimentos (DOE), por medio de superficies de respuestas se estimó un modelo del comportamiento en equilibrio termodinámico que se utilizará como referencia en futuros sistema de control.
\end{abstract}

Palabras clave: Adsorción, carbón activado, colector solar, metanol, refrigeración.

\section{ABSTRACT}

This work presents a characterization of a cooling system using solar radiation as the only source of energy to generate the adsorption phenomenon assisted in a stationary flat plate collector. This collector has an area of $1.1 \mathrm{~m}^{2}$ working with activated charcoal as adsorbate and using methanol as an adsorbent. The experimental results were compared in two conditions of tests with the objective to cool two liters of water. Temperatures of generation were recorded in the solar collector above $50{ }^{\circ} \mathrm{C}$ for solar radiation above $800\left[\mathrm{~W} / \mathrm{m}^{2}\right]$. The COP of the system presented values above 0.4, according to isobaric cooling and adsorption stages. Measurements of solar radiation, temperatures of the system were performed, resulting in temperature differences of up to $8.6^{\circ} \mathrm{C}$ between the ambient temperature and the cold room. Local radiation samples of up to $1155\left[\mathrm{~W} / \mathrm{m}^{2}\right]$ were obtained, showing efficiency

1 Universidad Francisco de Paula Santander. Avenida Gran Colombia \#12E-96 Barrio Colsag, Cúcuta, Colombia. email: femgarcia@ufps.edu.co

2 Universidade Federal de Uberlandia. Avenida Joao naves de Ávila, 2121 Bairro santa Mónica, Uberlandia, Minas Gerais. Brasil. email: bandarra@ufu.br

3 Universidad Francisco de Paula Santander. Avenida Gran Colombia \#12E-96 Barrio Colsag, Cúcuta, Colombia. email: dinaelgi@ufps.edu.co

Autor de correspondencia: femgarcia@ufps.edu.co 
of the collector up to $75 \%$, at specific periods and $35 \%$ at total daily efficiency. Using the Design of Experiments (DOE), through response surfaces, a model of behavior in thermodynamic equilibrium to be used as a reference in futures control systems.

Keywords: Adsorption, activated carbon, solar collector, methanol and refrigeration.

\section{INTRODUCCIÓN}

En la región nororiental de Colombia en nuestro caso, donde el calor es abundante y el sol es nuestra compañía diaria, el vivir se hace difícil en el casco urbano a pesar de que se cuenta con sistemas convencionales de refrigeración por compresión, sin embargo, en los linderos de la ciudad por no ir muy lejos, no se cuenta con un servicio eléctrico bueno y por lo tanto se hace difícil el uso de sistemas de aire acondicionado, sumado a esto la necesidad no solo de refrescar ambientes, sino el mantener refrigerado alimentos perecederos, artículos farmacéuticos, etc. Y sin mencionar que es prioritaria la disminución de la huella de carbono que estamos dejando desde nuestros hogares, no es solo responsabilidad de las grandes empresas, desde nuestras casas es vital hacer estos esfuerzos.

Como solución a estos problemas, de refrigeración, solución energética y disminución de la huella de carbono, diversos centros de investigación no se han ahorrado esfuerzos en estudios de sistemas de refrigeración que usen fuentes de energía alternativas, [4] por ejemplo con sistemas de refrigeración por adsorción usando energía solar, bien sea con paneles fotovoltaicos o por energía solar térmica, donde en lugar de usar los sistemas de vapor compresión, aunque no sean lo suficientemente robustos para refrigerar una sala, se ha visto que se puede alcanzar a congelar ciertas cantidades de agua, [18-19], ayudando a refrigerar alimentos perecederos y vacunas, mejorando así la calidad de la vida de los habitantes de la región. Los sistemas de refrigeración por adsorción solar, no emiten gases contaminantes a la atmosfera, y su elaboración es de fácil mantenimiento y algunos prototipos se pueden hacer con elementos de fácil adquisición.

El objetivo principal de este proyecto es fortalecer el desarrollo de futuros procesos de refrigeración para nuestra región asistidos por colectores solares de forma que se aproveche el fenómeno de adsorción con la promisoria en busca de optimizar la calidad de vida y el impacto al medio ambiente.

\section{CICLO REFRIGERACIÓN POR ADSORCIÓN}

Entre los sistemas de refrigeración térmica se encuentran los sistemas de refrigeración por adsorción solar y absorción solar, estos sistemas consiguen generar frio a partir del calor, y de las propiedades de algunas sustancias en retener ciertos componentes entre otros, la diferencia radica en que para la absorción hay una mezcla entre los pares absorbato-absorbente, lo que requiere una gran energía para su regeneración, mientras que la adsorción, retiene los componentes en la superficie de un sólido, Figura 1.

Fuente: [21].

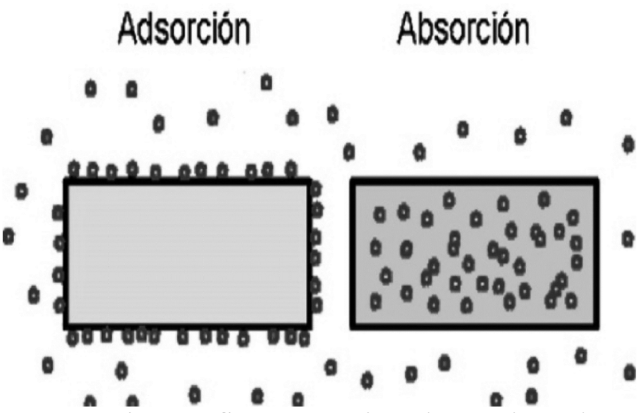

Figura 1. Representación gráfica entre adsorción y absorción.

La generación de frio por adsorción es una técnica promisoria para el campo de la refrigeración. Se basa en la generación de frio a partir de un consumo de calor, aportado por un fluido a una temperatura dada (para rendimientos aceptables en industria la temperatura mínima es de $90^{\circ} \mathrm{C}$ ) [4].

Este proceso puede ser visto de forma más clara en un diagrama de Clapeyron, mostrado en la Figura 2. El proceso comienza con una ganancia de calor a temperatura contaste de 1-2 en este punto el refrigerante es adsorbido por el adsorbente. La energía total ganada por el sistema durante el periodo de calefacción es la suma de energía desde el punto 1 al punto 2 y la energía del punto 2 al punto 3. La energía de 1 a 2 es la que se necesita para 
ascender la temperatura del adsorbente y refrigerante, y la energía de 2 a 3 es la energía requerida para la calefacción progresiva del adsorbente y para el proceso de desorción.

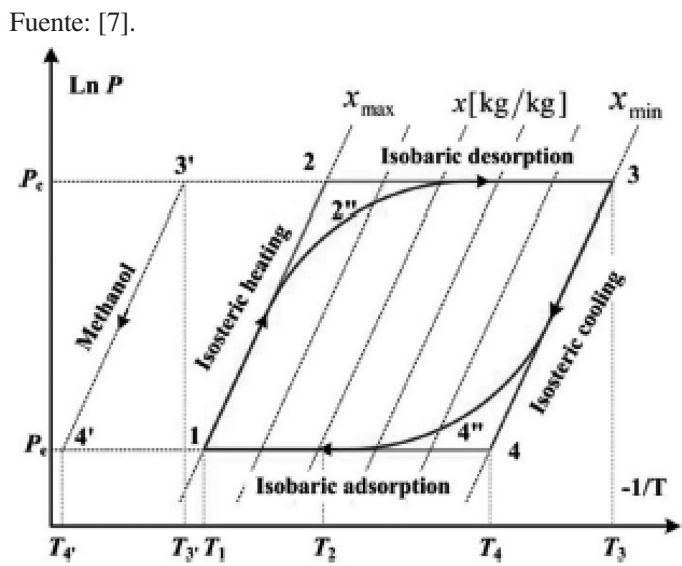

Figura 2. Diagrama Clapeyron ciclo ideal refrigeración por adsorción.

\section{DESCRIPCIÓN DE PROTOTIPO EXPERIMENTAL}

Como primera fase de nuestro proyecto de refrigeración fue importante la elección y construcción de un buen colector. En nuestro caso, apoyados por experiencias se construyó un colector de placa plana, para trabajar en rangos de temperaturas entre los $30^{\circ} \mathrm{C}$ y $80^{\circ} \mathrm{C}$. Dicho rango de temperatura fue posible alcanzarla a pesar del porcentaje de radiación total emitida por el sol que llega a nuestra superficie, que en un caso ideal es del $47 \%$ de forma directa y también se recomienda para este tipo de colectores usar un ángulo de inclinación que coincida con el ángulo de latitud por su cercanía con la línea del ecuador [11-12]. A continuación se presenta en la Figura 3a el diagrama P\&ID de sistema experimental construido.

Fuente: Autores.

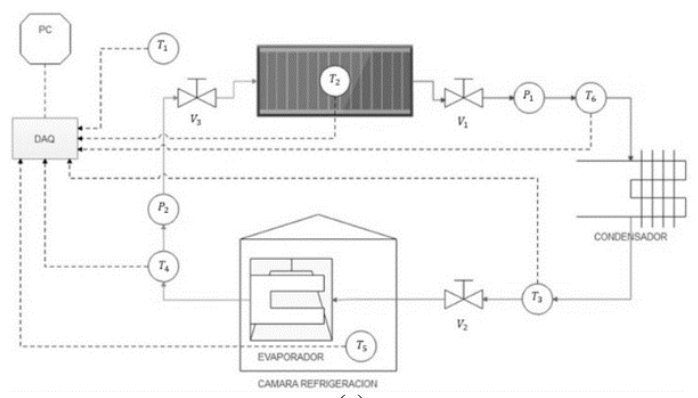

(a)

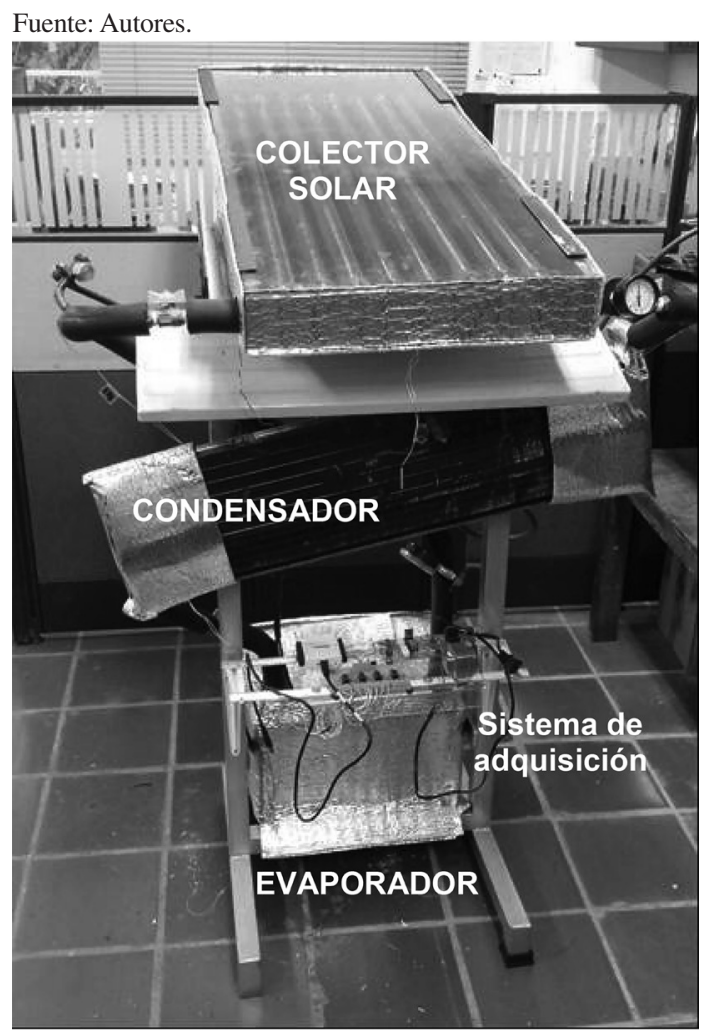

(b)

Figura 3. (a)Diagrama esquemáticoP\&ID experimental en la presente investigación. (b) Prototipo final del proyecto.

En la Figura 3 b se presenta la versión final del colector solar que asistió al sistema de refrigeración por adsorción construido. Dicho colector fue construido para un área de captación de $1,1 \mathrm{~m}^{2}$, totalmente aislado el cual contiene 8 tubos de material galvanizado de 1" concéntricos con 8 tubos de cobre con un diámetro de $1 / 2$ " y perforaciones de $2 \mathrm{~mm}$ distribuidas uniformemente a los largo de dichos tubos. Estas perforaciones tienen como función permitir el filtrado del absorbente metanol entre los $2 \mathrm{Kg}$ de carbón activado tipo CNR 115 distribuido uniformemente en los ochos tubos de 1".

Con respecto a los demás componentes del sistema de refrigeración, para el intercambiador condensador tipo aleta fue construido para un área de transferencia con el ambiente de 0,206 $\mathrm{m}^{2}$ asumiendo una eficiencia del $70 \%$. Dicha área permitió asegurar una condensación completa del metanol en 2,5 horas. Para el intercambiador evaporador. Este intercambiador fue diseñado 
para permitir la evaporización del metanol en 4 horas, cuya construcción a partir de tubos de cobre de $1 / 2$ " fue 10,9 metros de largo distribuido en 6 secciones de $30 \mathrm{cms}$. El evaporador fue inmerso en una caja térmica, totalmente aislada, la cual contiene la cantidad de agua que será el objetivo de esta primera fase a ser enfriada por dicho sistema de refrigeración, ver Figura 3b. Con respecto a la instrumentación, fueron instalados 6 sensores de temperatura tipo PT100 y dos medidores de presión tipo bourdon en nuestro banco experimental, según diagrama esquemático figura $3 \mathrm{a}$.

La curva de calibración para los sensores de temperatura se presenta en la Figura 4. Para el acondicionamiento de estos sensores se utilizaron circuitos amplificadores mediante integrados TL084, este integrado maneja 4 amplificadores operacionales. Para cada uno de los sensores de temperatura se necesitaron dos etapas de amplificación cuyo acondicionamiento se trabajó en rango de 0-5 Vdc como voltaje de salida, debido que este rango de voltaje es permitido por la tarjeta de adquisición DAQ. Dicha tarjeta DAQ modelo NI-6008 permitió implementar un sistema de supervisión online en software Labview ${ }^{\circledR}$ para monitoreo de todas la variables de estudio del banco experimental.

Fuente: Autores.

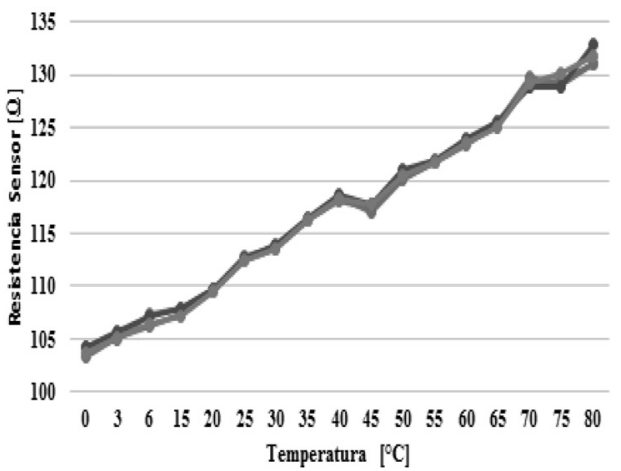

Figura 4. Curvas de calibración para los sensores de temperatura.

La incerteza de los parámetros calculados fue determinada según tratamiento estadístico experimental según [13] con un intervalo de confianza del $95 \%$. La exactitud de las mediciones y parámetros calculados se muestran en la Tabla 1.
Tabla 1. Exactitud de las mediciones y parámetros con un intervalo de confianza de $95 \%$

\begin{tabular}{|c|c|}
\hline Parámetro & Incerteza \\
\hline Temperatura $(T)$ & $\pm 1^{\circ} \mathrm{C}$ \\
\hline Radiación solar $\left(I_{r a d}\right)$ & $\pm 10 \mathrm{~W} / \mathrm{m}^{2}$ \\
\hline Humedad relativa $($ RH $\%)$ & $\pm 5 \%$ \\
\hline Coeficiente rendimiento $(\mathrm{COP})$ & $\pm 0,06$ \\
\hline Capacidad de refrigeración $\left(Q_{e v}\right)$ & $\pm 0,15$ \\
\hline
\end{tabular}

\section{RESULTADOS EXPERIMENTALES}

Los resultados reportados han sido obtenidos a partir de una serie de baterías experimentales realizadas el 17 de agosto y el 1 de septiembre de 2016 respectivamente, cuyas condiciones climáticas fueron diferentes (con alta radiación solar y otra condición cuando el día ha estado nublado representando baja radiación solar). Un primer análisis, trabajando el prototipo en forma manual, se indujo a una primera etapa de calentamiento isostérico desde las 7:00 hasta las 10:00 horas cerrando las válvulas del sistema $\left(V_{1}, V_{2}\right.$ y $\left.V_{3}\right)$, ver diagrama esquemático $\mathrm{P} \&$ ID de la figura $3 \mathrm{a}$. A partir de las 10:00 horas se abrió solamente la válvula $\left(V_{l}\right)$ cuyas condiciones a nivel de radiación solar fueron favorables al presentarse máximos en el orden de los 1150 [watts $/ \mathrm{m}^{2}$ ] para el 17 de agosto comparado con las pruebas del 1 de septiembre cuya máximo alcanzado fue 634 [watts $/ \mathrm{m}^{2}$ ], Figura $5 \mathrm{a}$. Estos picos en la radiación permitieron al colector solar, desarrollar el proceso de calentamiento isostérico y de desorción con temperaturas alcanzadas en el orden de $\operatorname{los} 80\left[{ }^{\circ} \mathrm{C}\right] \mathrm{y}$ los $60\left[{ }^{\circ} \mathrm{C}\right]$ respectivamente, Figura $5 \mathrm{~b}$.

La Figura 5a, constata según el principio de Luis Jutglar sobre la declinación solar entre la línea Sol-Tierra y el plano ecuatorial celeste que influye directamente la radiación recibida en la superficie [14], [15].

Fuente: Autores.

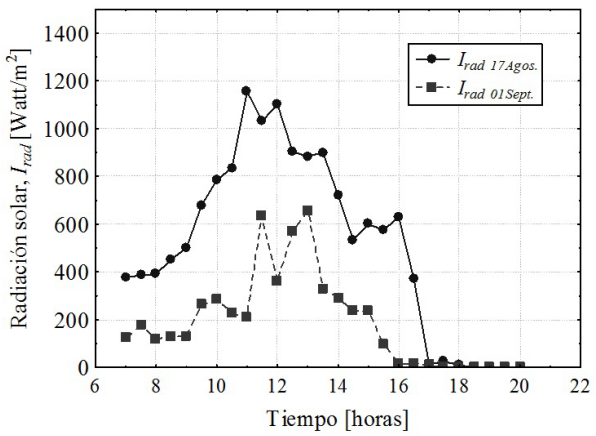

(a) 


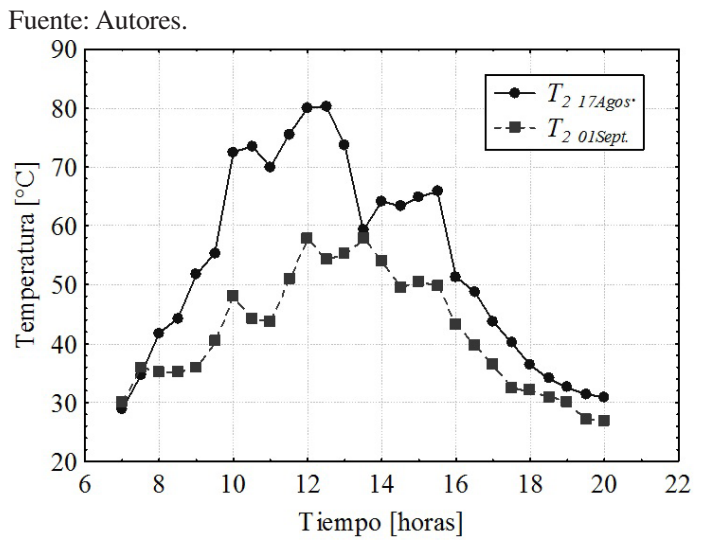

(b)

Figura 5. (a) Radiación solar $\left(I_{\text {rad }}\right)$. (b) temperatura interna colector $\left(T_{2}\right)$.

Este sistema de refrigeración está siendo estudiado como intermitente y manual, es así, se busca asegurar que el carbón activado dentro de los tubos del colector por efecto de la energía solar inicie el proceso de desorber el metanol que está siendo liberado en vapor saturado. El hecho de alcanzar temperaturas internas del colector $\left(T_{2}\right)$ de $80^{\circ} \mathrm{C}$ se asegura que el intercambiador condensador reciba parte del vapor saturado de metanol desprendido para que este intercambie calor con el ambiente. Hasta ese momento el rendimiento del sistema COP es muy mínimo porque prácticamente el sistema se está cargando de energía desde el reservorio más caliente.

Fuente: Autores

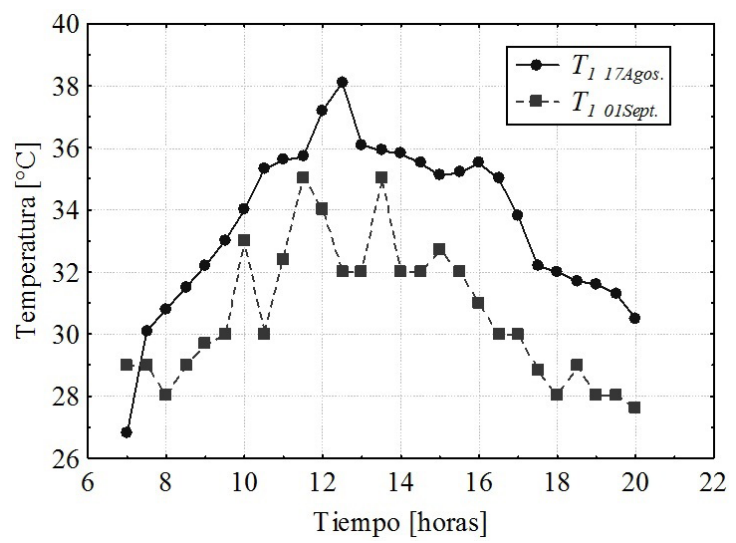

(a)

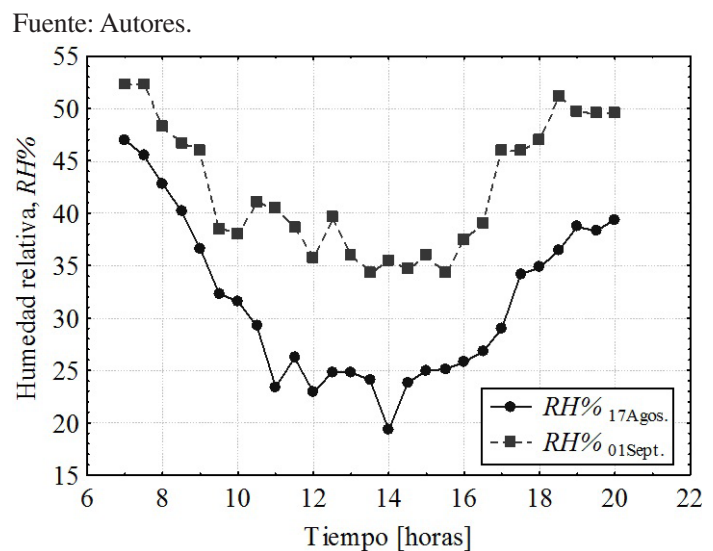

(b)

Figura 6. (a)Temperatura del ambiente $\left(\mathrm{T}_{1}\right),(\mathrm{b})$ Humedad relativa (RH\%).

Se registraron temperaturas del ambiente en el orden $38,1\left[{ }^{\circ} \mathrm{C}\right]$ como máximo y mínima de $26,8\left[{ }^{\circ} \mathrm{C}\right]$ a las 12:00 horas y a las 7:00 horas para el 17 de agosto. Sin embargo para el día 1 de septiembre se caracterizó por ser un día opaco cuya temperatura del ambiente no superó los $35\left[{ }^{\circ} \mathrm{C}\right]$, Figura $6 a$. Las humedades relativas presentaron comportamientos similares cuyos valores mínimos fueron identificados entre las 12:00 y 14:00 horas, que posteriormente se incrementan para la jornada tarde-noche como se puede observar en la Figura 6b. Para climas húmedos resulta mucho más apropiado indicar el grado de calor presente en la zona, al tratar en conjunto la temperatura ambiente con la humedad relativa. La sensación máxima de calor en los horarios del medio día se ve influenciado por los mínimos valores en la humedad relativa de la región. Ya en la jornada nocturna la humedad se va incrementando lo que permite tener sensaciones térmicas agradables cuando la temperatura del ambiente se comporta inversamente proporcional.

Cuando el sistema se induce manualmente a la etapa de enfriamiento isostérico después de las 14:00 horas abriendo la válvula $\left(V_{2}\right)$ y manteniendo las demás válvulas $\left(V_{1} y V_{3}\right)$ cerradas. Se buscó que el metanol fuese condensado en dos horas aproximadamente cuando fuese pasando por el condensador y por efecto de la gravedad llegue al evaporador.

Es así, después de las 16:00 horas la sección de la recamara aislada térmicamente donde se encuentra 
el evaporador empieza a recibir el metanol en fase liquida. El rendimiento (COP) del sistema se va mejorando después de las 16:30 horas, debido a la generación de un delta de temperatura en el agua contenida en dicha recamara $\left(T_{5}\right)$ referenciada con la temperatura del ambiente $\left(T_{l}\right)$, ver Figura 7. Prácticamente el sistema inicia un mejoramiento en su capacidad de refrigeración $\left(Q_{e v}\right)$ inducido por el manejo de las válvulas que aseguran que el fluido de trabajo en nuestro caso el metanol, pase por los diferentes estado en su cambio de fase y se vaya acumulando poco a poco en el evaporador. Un máximo de $\left(Q_{e v}\right)$ se experimenta en el orden de los 15 [watts] después de las 19:00 horas cuando el sistema inicia el proceso de evaporización del metanol en su totalidad, ósea etapa de adsorción del metanol al abrir la válvula $\left(V_{3}\right)$ y manteniendo las demás válvulas cerradas y así permitir el retorno del metanol hacia el colector solar. Dicho retorno es debido a que el metanol en su totalidad al evaporizarse en la recamara absorbe el calor del agua contenida. Se registra un enfriamiento en la temperaturas del agua $\left(T_{5}\right)$ en el orden de los $18\left[{ }^{\circ} \mathrm{C}\right]$ cuando la temperatura del ambiente está por encima de los $30\left[{ }^{\circ} \mathrm{C}\right]$. Mayores deltas de temperaturas $(\Delta T)$ fueron alcanzadas para las pruebas del día 1 septiembre en la jornada de la noche en comparación con las pruebas del 17 de agosto, ver Figura 7.

Fuente: Autores.

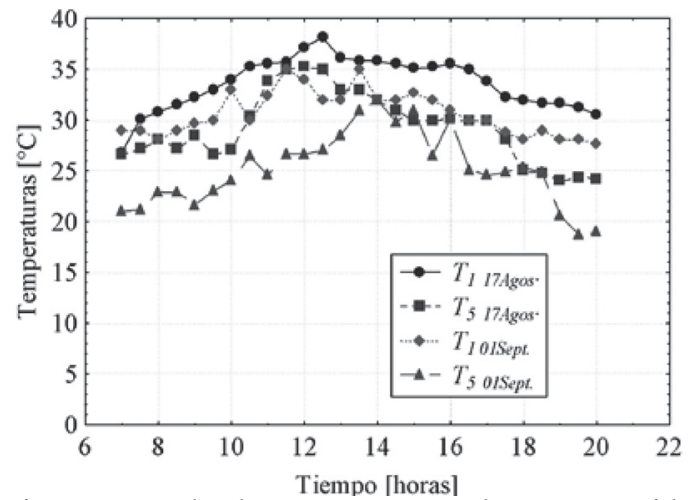

Figura 7. Delta de temperatura en el agua contenida en dicha recamara $\left(T_{5}\right)$ referenciada con la temperatura del ambiente $\left(\mathrm{T}_{1}\right)$.

El rendimiento del sistema está ligado y referenciado por el buen uso de las válvulas internas del sistema $\left(V_{1}, V_{2}, V_{3}\right)$. De forma que se asegura de acuerdo al transcurrir del día generar un frio por el cambio de fase que sufre el metanol a medida que pasa por los diferentes componentes que hacen parte de dicho sistema.
El colector solar trabaja como un compresor de forma natural aprovechando la capacidad del carbón y el metanol al adsorberse entre sí, por el efecto solar. Por ser un ciclo intermitente y manual, el sistema no rinde en sus etapas de desorción. En la siguiente tabla se presentan los principales parámetros de estudio del sistema de refrigeración bajo en equilibrio termodinámico con las condiciones de trabajo presentes los días 17 de agosto y 1 de septiembre.

Tabla 2. Comparativo parámetros de estudio sistema refrigeración solar. Fuente: autores.

\begin{tabular}{|c|c|c|}
\hline & $\mathbf{1 7}$ de agosto & 1 de septiembre \\
\hline $\boldsymbol{T}_{\boldsymbol{1} \text { máxima }}$ & $38,1^{\circ} \mathrm{C}$ & $35^{\circ} \mathrm{C}$ \\
\hline $\boldsymbol{T}_{2 \text { máxima }}$ & $80,3^{\circ} \mathrm{C}$ & $57,7^{\circ} \mathrm{C}$ \\
\hline $\boldsymbol{C O P}$ & 0,25 & 0,82 \\
\hline $\boldsymbol{P}_{\boldsymbol{I}_{\text {máxima }}}$ & $32,81 \mathrm{kPa}$ & $26,32 \mathrm{kPa}$ \\
\hline$\Delta \boldsymbol{T}_{\text {máxima }}$ & $6,3{ }^{\circ} \mathrm{C}$ & $8,6^{\circ} \mathrm{C}$ \\
\hline $\boldsymbol{I}_{\text {rad máxima }}$ & $1155 \mathrm{~W} / \mathrm{m}^{2}$ & $655 \mathrm{~W} / \mathrm{m}^{2}$ \\
\hline $\boldsymbol{T}_{5}$ & $24,2^{\circ} \mathrm{C}$ & $19^{\circ} \mathrm{C}$ \\
\hline$\eta_{\text {colector }}$ & $39.7 \%$ & $10,2 \%$ \\
\hline
\end{tabular}

Con los datos adquiridos se procedió a generar superficies de respuesta DOE (Desing of the experiment), dichas superficies representan modelos teóricos que caracterizaran la naturaleza fundamental del prototipo. Fueron definido como factores de control la presión $\left(P_{l}\right)$ en el colector solar y nuestra radiación solar $\left(I_{\text {rad }}\right)$; por otro lado, las variables como el COP, $\left(Q_{e v}\right), T_{5}$ son variables dependientes, y cualquier cambio presente en $\left(P_{1}\right)$ e $\left(I_{\text {rad }}\right)$ afectan directamente las variables dependientes. Esta clasificación se sustenta en el hecho de que nos permita a futuro desarrollar estrategias de control en la presión $\left(P_{l}\right)$ a partir del ajuste la abertura de la válvula $\left(V_{l}\right)$ cuya finalidad en optimizar la eficiencia del colector ante la gran variabilidad de las condiciones climáticas día a día en nuestra región.

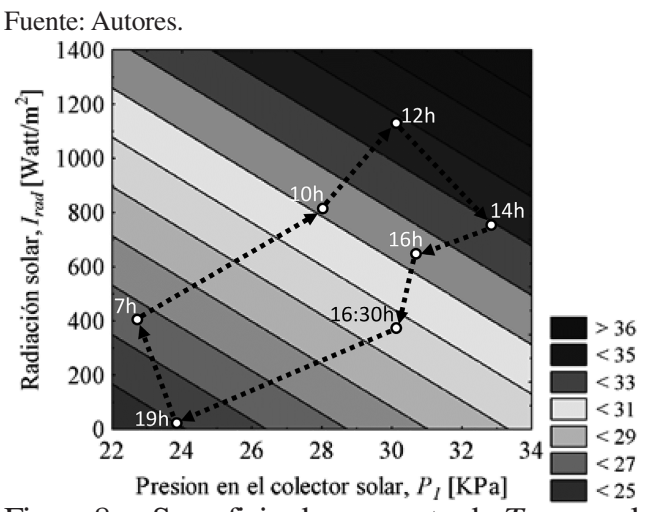

Figura 8. Superficie de respuesta de $T_{5}$ con valores medidos en el transcurso del día. 
En la Figura 8 podemos observar que a medida que baja la radiación y la presión en el colector, la temperatura $T_{5}$ comienza a descender, esto es característico de los sistemas de refrigeración por adsorción intermitentes, donde solo se observa refrigeración en horas de la noche, podemos constatar en la presente investigación y en trabajos como [20], [4] entre otros; donde se encontraron que las temperaturas en el evaporador más bajas fueron en horas de la noche, en horas del día donde la temperatura y presión aumentan, la temperatura en el evaporador también aumentaba. Realizando los análisis respectivos se obtuvo una función objetivo basada en información del sistema en equilibrio termodinámico.

$$
T_{5}=14,537+0,433 P_{1}+0,0055 I_{\text {rad }}
$$

Esta función objetivo que se toma en equilibrio termodinámico, fue evaluada y comparada con los datos medidos de manera experimental. En la Figura 9, podemos observar la estimación según modelo $D O E$ cercanos a los valores tomados en las pruebas experimentales. Demostrando que el modelo satisface experimentalmente con una correlación del $89 \%$.

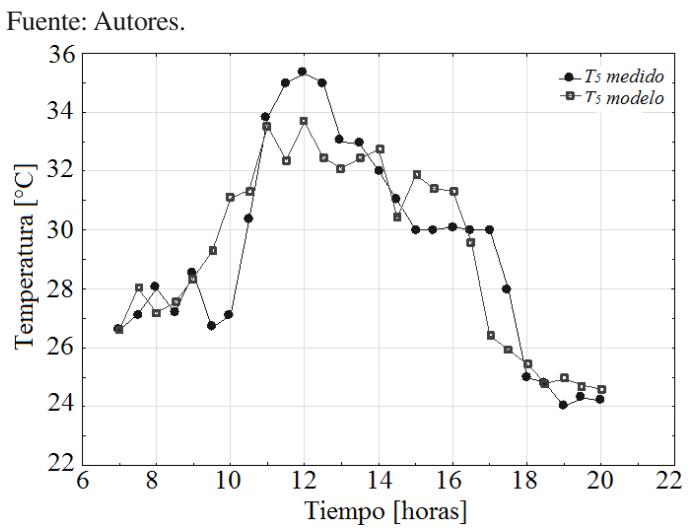

Figura 9. Comparación respuesta temperatura $\left(T_{5}\right)$ según estimación modelo obtenido vs $\left(T_{5}\right)$ mediciones experimentales.

En la Figura 10 se puede observar que a medida que la radiación disminuye la capacidad de enfriamiento mejora, el calor en nuestro colector disminuye y por lo tanto se reduce la presión en nuestro sistema es allí donde observamos que mejora nuestra capacidad de enfriamiento.

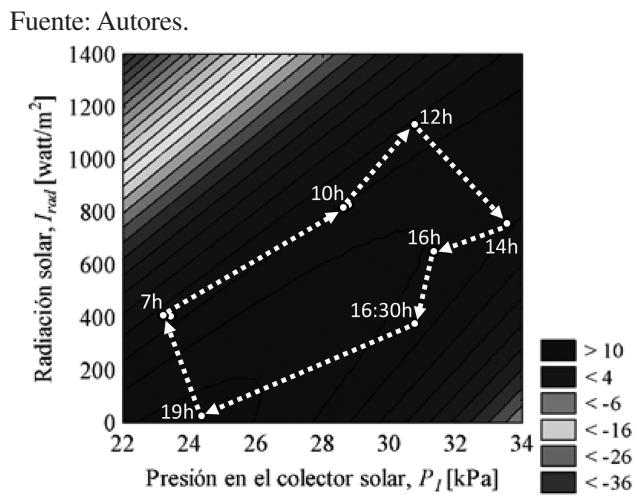

Figura 10. Superficie de respuesta de la capacidad de enfriamiento con valores medidos en el transcurso del día.

\section{CONCLUSIONES}

El diseño y estudio de este prototipo fue en estado estable como parte inicial de una línea de investigación que busca alternativas en el campo de la refrigeración a futuro. De tal forma se obtuvieron resultados satisfactorios que permitieron confirmar la posibilidad de lograr enfriar una cantidad de agua en una recamara aislada sin necesidad de equipos conectados a la red eléctrica, como es el caso de los sistemas de refrigeración convencional.

Siguiendo la literatura [16], [17], se concluye que la eficiencia del colector solar para el día 17 de agosto estuvo entre el $22.2 \%$ al $59.2 \%$ y una eficiencia promedio diaria de 39,74\%; para el día 1 septiembre se presentaron valores muy mínimos de eficiencia por la presencia de lluvias y frentes de vientos fríos, sin embargo en puntos máximos alcanzó temperaturas por encima de $50\left[{ }^{\circ} \mathrm{C}\right]$. Esto nos permite a futuro centrarnos a estudiar la optimización de este tipo colector incorporando materiales PCM (phase change material) y volverlo más robusto a dichas variables externas.

El rendimiento térmico del sistema de refrigeración (COP) fue satisfactorio en un rango de 0,25 a 0,8 como máximo, comparado dentro de la literatura internacional [4], [5]. Sin embargo está muy por debajo de los convencionales sistemas de refrigeración por compresión.

$\mathrm{Al}$ ser un ciclo intermitente e inducido a trabajar manual, el buen uso de las válvulas asegura el cambio 
de fase del metanol por los diferentes componentes. Es así, se alcanzó temperaturas del agua contenida en la recamara por el orden de los $18\left[{ }^{\circ} \mathrm{C}\right]$ con una mínima cantidad de metanol de 1 litro.

Estos modelos son de utilidad para ser usados como patrón en un sistema de control, que pueden dictaminar el punto óptimo de operación del sistema. Con estos modelos nuestro sistema puede comparar los valores medidos y tomar la acción correctiva para poder llegar a la zona de trabajo optima del sistema, como futuros trabajos a nivel de control y optimización.

\section{AGRADECIMIENTOS}

Los autores gustan de expresar su agradecimiento a la vicerrectoría de investigación y extensión UFPS por su apoyo financiero a través convocatoria de proyectos FINU y logística [22].

\section{REFERENCIAS}

[1] H. Ammar, Benhaoua y Balghouthi, "Simulation of tubular adsorber for adsorption refrigeration system powered by solar energy in sub-Sahara region of Algeria". ScienceDirect, pp. 31-40, 2015.

[2] E.E. Anyanwu. "Review of solid adsorption solar refrigeration II: An overview of the principles and theory". ScienceDirect, pp. 1279-1295. 2004.

[3] L. Cardona y R. Rojas. "Diseño y construcción de un colector solar plano". Mexico. 2007.

[4] K. Edem, N’Tsoukpoe, D. Yamegueu y J. Bassole. "Solar sorption refrigeration in Africa". ScienceDirect, pp. 318-335. 2014.

[5] D. Bressan, F. Belo y A. Ferreira. "Ar condicionado solar por adsorção: fundamentos e estado da arte". Fortaleza: I Congresso Brasileiro de Energia Solar. 2007.

[6] R. Treybal. Operaciones de transferencia de masa, Rhode Island: McGrawHill.

[7] H.Ambarita and H. Kawai. "Experimental Study on Solar powered adsorption refrigeration cycle with activated alumina and activated carbon as adsorbent". ScienceDirect, pp. 36-46. 2016.

[8] M.Berdja, B.Abbad, F. Yahi, F. Bouzefour and M. Ouali. "Desing and realization of a solar adsorption refrigeration machine powered by solar energy". Science Direct, pp. 1226-1235. 2014.
[9] M.G. Campuzano Paéz y J.V. Chiriboda Albuja. "Diseño y construccion de un sistema de enfriamiento por adsorción solar". Quito: Escuela Politécnica Nacional. 2010.

[10] E. Hernandez Arroyo y M. A. Reyes Orozco. "Diseño construccion y prueba de un refrigerador por adsorcion solar, usando carbon activado". Bucaramanga: Universidad Pontificia Bolivariana. 2011.

[11] H. Grossi Gallegos y R. R. “Ángulo optimo para colectores planos de energía solar integrados a edificios". Avances en Energias Renovables y medio ambiente, pp. 7. 2012.

[12] K. Bouhadef, S. Chikh, A. Boumedien y A. Benabdesselam. "Desingand Analysis of Solar Refrigeration System with a Roating Generator". Internactional Journal of Mechanical, Aerospace, Industrial and Mechatronics Engineering, pp. 501 - 505. 2013.

[13] R. Abernethy and J. Thompson. Handbook Uncertainty in gas turbine measurements, Tennesse: AEDC. 1973.

[14] L. Jutglar. "Energia Solar". Barcelona. Ediciones Ceac. 2004.

[15] J. Duffie (Deseased) and W. Beckam. "Solar Engineering of Thermal Process". Wisconsin. Wiley. 2013.

[16] A.E. Ávila Gómez, M. Fandiño, J. Mario y J.F. Beltran Sarmiento. "Evaluación energética de un colector solar de placa plana de doble cubierta". Ingeniería y Desarrollo. Vol. 27, pp. 93-112. 2010.

[17] L. Berriz Perez y M. Alvarez Gonzalez. "Manual para el Calculo y Diseño de Calentadores Solares". Ciudad de la Habana. Cuba Solar. 2008.

[18] N. Abu-Hamdeh, K. Alnefaie and K. Almitani. "Desing and performance characteristics of solar adsorption refrigeration system using parabolic trough collector: Exprimental and statistical optimization technique". Energy Conversion and Management. Vol. 74, pp. 162 - 1170. 2013.

[19] E. Anyanwu and C. Ezekwe. "Desing, construction and test run of a solid adsorption solar refrigerator, using activated carbon/ methanol, as adsorbent/adsorbate pair". Energy Conversion \& Management. Vol. 44, pp. 2879-2892. 2003.

[20] P. Islam, T. Morimoto. "A new zero energy cool chamber with a solar driven adsorption refrigerator". Renewable Energy. Vol. 72, pp. 367-376. 2014. 
[21] K. Guillen, A. Perera y M. Aguilar. Procesos de adosrción. 17 de abril de 2015. Fecha de consulta: 5 de marzo de 2016. URL: http://fisicobloga.blogspot.com.co/2015/04/ procesos-de-adsorcion.html
[22] J.J. Silva Villamizar. "Diseño y construcción de un sistema de refrigeración por adsorción asistido a traves de un colector solar experimental". Universidad Francisco de Paula Santander. Tesis, pp. 115. 2016. 